\title{
Disseminated histoplasmosis diagnosed on routine outpatient endoscopy in a renal transplant patient
}

\author{
Sylvester Luu*1, Brian C. Benson², Jonathan W. Schwake² \\ ${ }^{1}$ Department of Internal Medicine, Brooke Army Medical Center, San Antonio, Texas, United States \\ ${ }^{2}$ Department of Gastroenterology, Brooke Army Medical Center, San Antonio, Texas, United States
}

Received: January 23, 2018

DOI: $10.5430 /$ crim.v5n3p1
Accepted: March 13, 2018

URL: https://doi.org/10.5430/crim.v5n3p1

\begin{abstract}
A 58-year-old female with prior renal transplant on immunosuppression underwent upper endoscopy and colonoscopy to evaluate iron deficiency anemia. Her upper endoscopy appeared normal and her colonoscopy showed mild colitis in her transverse colon. Biopsies of her duodenum, taken to evaluate for celiac disease, as well as biopsies of the colitis showed intracellular yeast within histiocytes in the lamina propria consistent with disseminated histoplasmosis. This case describes a renal transplant patient with disseminated gastrointestinal histoplasmosis $(\mathrm{GIH})$, which is rarely diagnosed on routine outpatient endoscopy.
\end{abstract}

Key Words: Histoplasmosis, Endoscopy, Renal transplant

\section{INTRODUCTION}

Histoplasmosis is an endemic mycosis that primarily infects the lungs and is often asymptomatic. In people with suppressed immunity, hematogenous dissemination can occur and, if left untreated, is progressive and fatal. The most common site of dissemination is the gastrointestinal tract, which often leads to gastrointestinal symptoms and is rarely found incidentally on routine outpatient endoscopy. Described is a case of disseminated gastrointestinal histoplasmosis (GIH) in a renal transplant patient diagnosed on routine outpatient endoscopy.

\section{CASE Presentation}

A 58-year-old female was referred to gastroenterology for iron deficiency anemia. She had been started on parenteral iron therapy by her nephrologist and had normal iron stores at the time of her evaluation. She reported several years of epigastric burning and abdominal bloating attributed to functional dyspepsia which had previously been evaluated with an abdominal ultrasound and hepatobiliary scintigraphy. She denied diarrhea, melena and hematochezia. Medical history was pertinent for hypertension, chronic kidney disease, and prior renal transplant in 1996 secondary unknown etiology; differential to include chronic glomerulonephritis versus hypertensive nephrosclerosis. Initial transplant was lost to chronic allograft nephropathy and she underwent a second cadaveric renal transplant in June 2013. Her immunosuppression regimen included mycophenolate mofetil $500 \mathrm{mg}$ BID, tacrolimus $2.5 \mathrm{mg}$ BID, and prednisone $5 \mathrm{mg}$ daily. However, in 2014, patient had biopsy confirmed acute antibody-mediated rejection resulting in loss of her second transplant. Presently, she is not on any immunosuppressive therapy. Her last colonoscopy was 10 years prior and was reportedly normal. She was referred for upper endoscopy and colonoscopy to further evaluate her iron deficiency. On endoscopy, the duodenum appeared normal with random biop-

\footnotetext{
*Correspondence: Sylvester Luu; Email: sylvesterluu@gmail.com; Address: Department of Internal Medicine, Brooke Army Medical Center, San
} Antonio, Texas, United States. 
sies taken to evaluate for celiac disease. Her colonoscopy was notable for mild colitis in the transverse and descending colon with a mild nodular appearance and friability (see Figure 1). Biopsies showed chronic active colitis with abundant intracellular fungal elements (see Figure 2). The GomoriGrocott methenamine silver (GMS) stain showed intracellular yeast forms within histiocytes in the lamina propria (see Figure 3 ) in both the duodenum and more diffusely in the colon, consistent with disseminated histoplasmosis. She was admitted to the hospital and started on itraconazole therapy. Her dyspepsia symptoms persisted despite several months of antifungal therapy. Patient completed a yearlong course of oral itraconazole therapy with resolution of symptomology and anemia with adequate iron store levels. However, on February 2018, patient resumed oral itraconazole therapy in the setting of incidentally noted positive urine histoplasmosis antigen while undergoing treatment for Candida Glabratta associated hematuria.

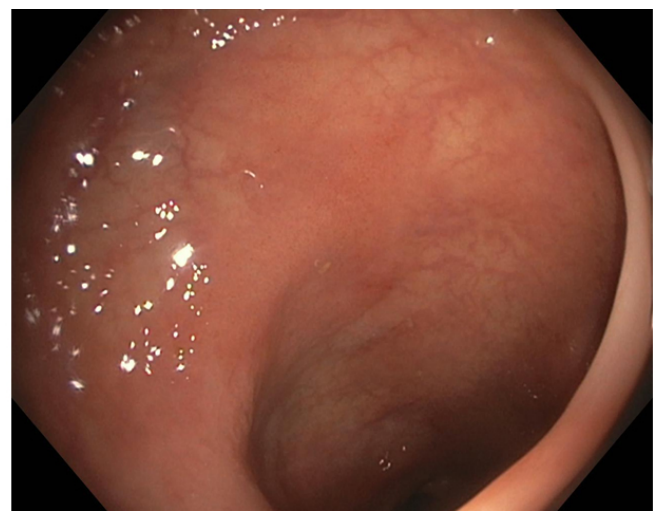

Figure 1. Mild colitis in the transverse and descending colon with mild nodularity and friability

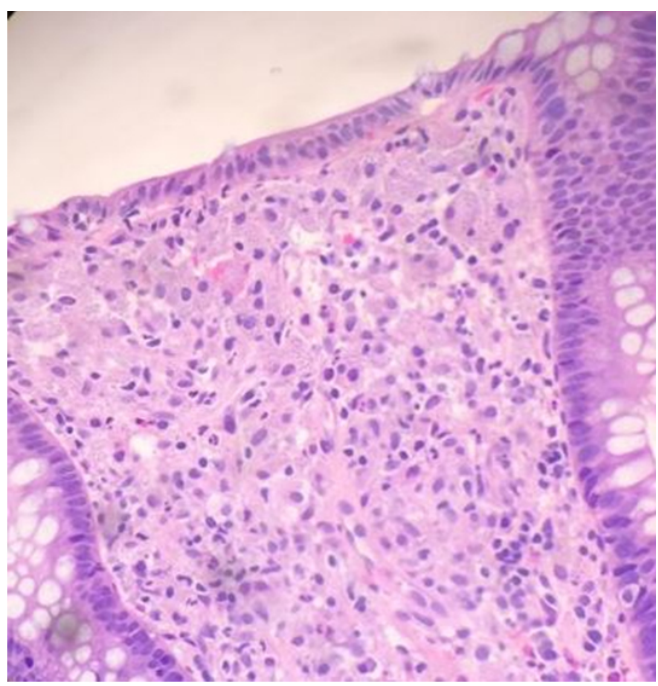

Figure 2. H\&E stain demonstrating chronic active colitis with abundant intracellular fungal elements

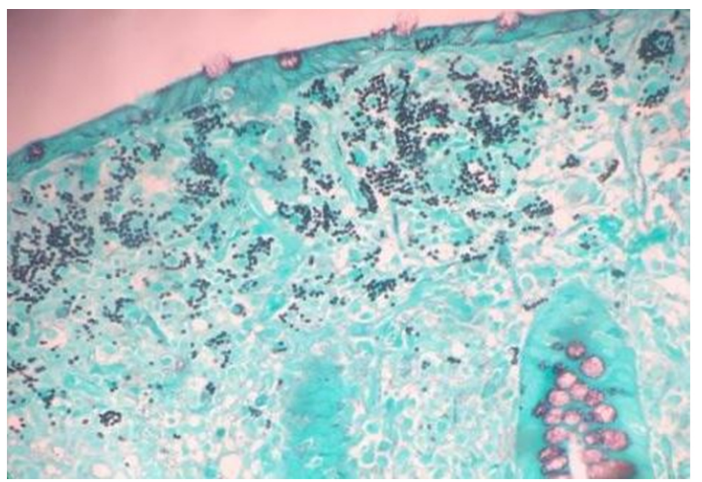

Figure 3. Gomori-Grocott methenamine silver (GMS) stain demonstrating intracellular yeast forms within histiocytes in the lamina propria

\section{Discussion}

Histoplasma capsulatum is a fungal pathogen endemic to the river valleys of Ohio, Missouri, and Mississippi and primarily infects the lungs. ${ }^{[1]}$ Most lung infections do not cause symptoms, but may lead to pneumonia, lung nodules, cavitary lesions and more rarely hematologic dissemination. Immunocompromised individuals, particularly those with AIDS or history of solid organ transplant, have the greatest risk of developing disseminated histoplasmosis. ${ }^{[2,3]}$ Disseminated histoplasmosis most commonly affects the gastrointestinal tract, but may also disseminate to other organs to include the skin, adrenal gland, central nervous system, lymph nodes, liver, spleen, and bone marrow. ${ }^{[4]}$

GIH is generally asymptomatic and is rarely diagnosed on routine outpatient endoscopy. When present, nonspecific symptoms of GIH include fever, weight loss, abdominal pain, diarrhea, nausea, anorexia, vomiting, and constipation. ${ }^{[4,5]}$ Gastrointestinal involvement in disseminated histoplasmosis is recognized in less than $10 \%$ of patients. At autopsy, however, it is seen in $70 \%$ of those with disseminated histoplasmosis, suggesting GIH has a higher prevalence than is clinically recognized. ${ }^{[6,7]}$

GIH can be found anywhere along the GI tract, from mouth to anus, however the highest incidence is within the ileum and cecum. ${ }^{[5]}$ It is suspected that the abundance of gutassociated lymphoid tissues, such as Peyer's patches in the terminal ileum, allow entry of infected macrophages. The duodenum is rarely involved, appreciated in less than $4 \%$ of cases, making it one of the rarest sites of GI involvement. On endoscopy, these lesions may appear inflamed, thickened, ulcerated, stricture, polypoid or tumor-like. ${ }^{[5]}$ However, in this case, the duodenum appeared endoscopically normal and biopsies were taken only as part of her iron deficiency evaluation. 
This case describes an unexpected diagnosis of disseminated histoplasmosis in a renal transplant patient diagnosed on routine outpatient endoscopy for evaluation of iron deficiency anemia. The only other reported cases of intestinal histoplasmosis in the literature are in the setting of intestinal perforation or GI bleeds. ${ }^{[3,8,9]}$ It is prudent that gastroenterologists, along with transplant nephrologists and transplant infectious disease specialists to be aware that GIH can be incidentally found on endoscopy and if left untreated, it is progressive and fatal over the course of weeks to years. Immunosuppressed individuals, particularly those with AIDS or organ transplantation, are at greatest risk. Standard treatment consists of two weeks of intravenous amphotericin B deoxycholate followed by an azole typically resulting in good clinical response. ${ }^{[10,11]}$

\section{CONFlicts of InTEREST Disclosure}

The authors declare that they have no competing interests.

\section{Consent}

Informed consent has been obtained from the patient for publication of the case details in this case report.

\section{Disclosures}

The view(s) expressed herein are those of the author(s) and do not reflect the official policy or position of Brooke Army Medical Center, the U.S. Army Medical Department, the U.S. Army Office of the Surgeon General, the Department of the Army, the Department of the Air Force and Department of Defense or the U.S. Government.

\section{REFERENCES}

[1] Mandell W, Goldberg DM, Neu HC. Histoplasmosis in patients with the acquired immune deficiency syndrome. Am J Med. 1986; 81(6): 974-8. https : //doi .org/10.1016/0002-9343(86) 90390-6

[2] Garcia RA, Jagirdar J. Colonic histoplasmosis in acquired immunodeficiency syndrome mimicking carcinoma. Ann Diagn Pathol. 2003; 7(1): 14-19. https://doi.org/10.1053/adpa.2003.50002

[3] Zainudin BM, Kassim F, Annuar NM, et al. Disseminated histoplasmosis presenting with ileal perforation in a renal transplant recipient. J Trop Med Hyg. 1992; 95(4): 276-9. PMid:1495124.

[4] Yang B, Lu L, Li D, et al. Colonic involvement in disseminated histoplasmosis of an immunocompetent adult: case report and literature review. BMC Infect Dis. 2013; 13: 143. https ://doi .org/10.1 186/1471-2334-13-143

[5] Assi M, McKinsey DS, Driks MR, et al. Gastrointestinal histoplasmosis in the acquired immunodeficiency syndrome: report of 18 cases and literature review. Diagnostic Microbiology and Infectious Disease. 2006; 55(3): 195-201. https://doi.org/10.1016/j.diag microbio. 2006.01.015

[6] Kahi CJ, Wheat LJ, Allen SD, et al. Gastrointestinal histoplasmosis. American Journal of Gastroenterology. 2005; 100(1): 220-231. https://doi.org/10.1111/j.1572-0241.2005.40823.x

[7] Suh KN, Anekthananon T, Mariuz PR. Gastrointestinal histoplasmosis in patients with AIDS: case report and review. Clinical Infectious Diseases. 2001; 32(3): 483-491. https ://doi.org/10.1086/31 8485

[8] Kourda N, Mlika M, Zidi YS, et al. [Cecal perforation in a kidney transplant patient: Disseminated histoplasmosis.] Med Trop (Mars). 2010; 70(5-6): 533-6.

[9] Syed TA, Salem G, Kastens DJ. Lower Gastrointestinal Bleeding Secondary to Intestinal Histoplasmosis in a Renal Transplant Patient. ACG Case Rep J. 2017; 4: e93. https://doi .org/10.14309/c rj.2017.93

[10] Wheat J, Sarosi G, McKinsey D, et al. Practice guidelines for the management of patients with histoplasmosis. Clin Infect Dis. 2000; 30: 688-695. https ://doi.org/10.1086/313752

[11] Wheat J, Connolly-Stringfield PA, Baker RL, et al. Disseminated histoplasmosis in the acquired immune deficiency syndrome: clinical findings, diagnosis and treatment, andreview of the literature. Medicine. 1990; 69(6): 361-374. https://doi.org/10.1097/00 005792-199011000-00004 